\title{
BVR CCD Photometric observation analysis of spiral galaxy Ic 467
}

\author{
Sinan H. Ali, Sundus A. Abdullah Albakri
}

Department of Astronomy and Space, College of Science, University of Baghdad

E-mail: sinanalbayati@yahoo.com

\begin{abstract}
The structure and composition of the stellar population in the surface brightness galaxy Ic 467 is studied using BVR CCD photometry. The observations were obtained on the $1.88 \mathrm{~m}$ optical telescope of Kottamia Astronomical Observatory, KAO, Egypt. A two-dimensional decomposition of the galaxy bulge and disk components is carried out. A powerful star forming region is observed near the galactic center. Based on the positions of the various components of the galaxy in two color diagrams and the surface brightness of the eastern arms in V filter is brighter than the western arm. From the observations, the surface brightness profiles, Ellipticity profiles, position angle profiles and color indices profiles are described and studied.
\end{abstract}

Key words

spiral galaxy, surface brightness, position angle, color index.

Article info.

Received: May. 2014

Accepted: Jun. 2014

Published: Sep. 2014

تحليل الارصاد الفوتومترية للمجرة الحلزونية (Ic 467) باستخدام كاميرا الشحن المزدوج بالمرشحات

(BVR)

$$
\text { سنان حسن علي, سندس عبد العباس البكري }
$$

الخلاصة

تم دراسة تركيب وبنية الجمهرة النجمية في اللمعان السطحي للمجرة (IC 467) بو اسطة أخذ أرصادات فوتومترية باستخدام

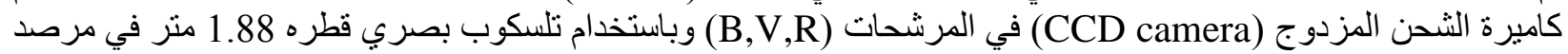

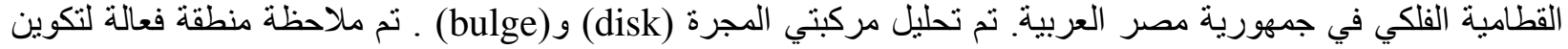

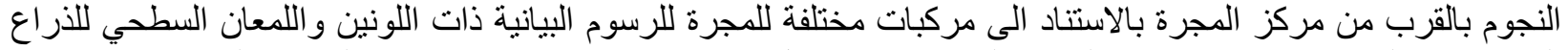

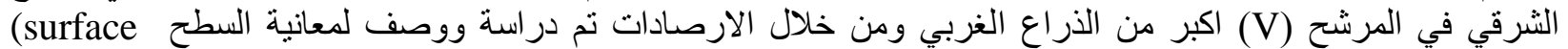

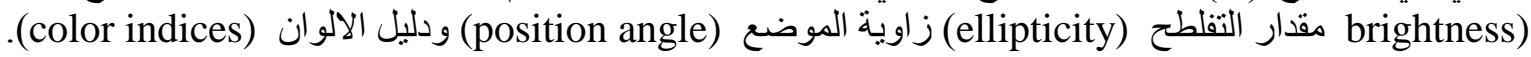

\section{Introduction}

Spiral galaxies consist of distinct building blocks; disk, bulge, and dark halo. The Hubble sequence is basically a sequence of the disk to bulge ratio which can be estimated by the surface photometry of galaxies. The galaxy has massive bulge whose brightness decreases accordance with a de Vaucouleurs law [1]. The goal of a surface photometry process to find out the structure of galaxies by morphological type[2]. Ic 467is among the most interesting nearby galaxies, and worthy of detailed study. It is a nearby, relatively small galaxy with a low surface brightness and two bright symmetrical spiral arms branch in the outer disk of Ic 467, forming several long extended arms [3]. Table 1 presents the basic parameters of the galaxy taken from NASA/IPAC EXTRAGALACTIC DATA BASE. 
Table 1: Basic parameters of the galaxy Ic 467 (NASA).

\begin{tabular}{|l|l|}
\hline Parameters & Ic 467 \\
\hline Galaxy type & SAB $(\mathrm{s}) \mathrm{c}$ \\
\hline RA & $07 \mathrm{~h} 30 \mathrm{~m} 18.4 \mathrm{~s}$ \\
\hline DEC & $+79 \mathrm{~d} 52 \mathrm{~m} 21 \mathrm{~s}$ \\
\hline Velocity km/s & 2042 \\
\hline Red shift & 0.006811 \\
\hline Apparent Magnitudes & 21.3 \\
\hline Surface brightness & 13.21 \\
\hline Position angle Diameter & $80^{\circ}$ \\
\hline $\begin{array}{l}\text { Major Diameter } \\
\text { (arcmin) }\end{array}$ & 1.3 \\
\hline $\begin{array}{l}\text { Minor } \\
\text { (arcmin) }\end{array}$ & \\
\hline
\end{tabular}

\section{Observations and data reduction} Ic 467 was observed on April 8-9, 2011, at the Newtonian focus of the $(1.88 \mathrm{~m})$ reflector telescope which has a focal length of $9.15 \mathrm{~m}$ at Kottamia Astronomical Observatory (KAO), Egypt. The photometric system of the CCD camera with $\mathrm{V}, \mathrm{R}$, and I broadband filters is close to the standard VRI Johnson-Cousins system. The size of the CCD is $1.024 \times 1.024$ pixels. The CCD was cooled with liquid nitrogen providing an image scale of 0.305arcsec/pixel [4]. An observing $\log$ is presented in Table 2.

Table 2: Log of observations.

\begin{tabular}{|c|c|c|c|c|}
\hline Date of observation & Filters & Time exposure $(\mathrm{sec})$ & Seeing & airmass \\
\hline $2011 / 4 / 8-9$ & $\mathrm{~B}$ & $5 \times 1200 \mathrm{sec}$ & $2.43^{\prime \prime}$ & 1.60 \\
& $\mathrm{~V}$ & $5 \times 1200 \mathrm{sec}$ & $2.57^{\prime \prime}$ & 1.64 \\
& $\mathrm{R}$ & $5 \times 1200 \mathrm{sec}$ & $2.68^{\prime \prime}$ & 1.63 \\
\hline
\end{tabular}

Five exposures were made in each filter. The duration exposures in each filter were 1200s. The seeing was 2.43" - 2.68".

The subsequent reduction of the data was carried out at Kottamia Astronomical Observatory (KAO), Egypt using the standard procedures in the Astronomical Image Processing Packages software IRAF (image-reduction system). The main reduction steps included flat fielding and correction for the CCD bias; removal of cosmic-ray traces; subtraction of the sky background for each image; superposition of the galaxy images using reference stars; addition of the galaxy images made in a single filter; corrections for differences of the instrumental photometric system from the standard Johnson-Cousins system and the air mass; and subtraction of the galaxy images made in different filters in order to obtain color-index maps[5]. To correct for atmospheric extinction, we used observations of the Landolt [6] standard stars SA 101-326 observed on the same night. The images of galaxies Ic 467 are recorded on CCD camera using $\mathrm{B}, \mathrm{V}$ and $\mathrm{R}$ filters. These images are illustrated in Fig.1. The principal reduction stages included the following [1,7-9].

(a) Correction of the data from the over scan region of the array.

(b) Subtraction of the bias from each frame of the data and flat field frames.

(c) Flat fielding the data frames.

(d) Determining the sky background and subtracting it from each image.

(e) Removal cosmic rays from the data frames.

(f) Correcting for the air mass.

(g) Combining of images made in the same filters to get the master frame.

(h) Photometric calibration was carried out to translate instrumental measurements to standard measurements based on the Landolt standard star is SA 101-326. 
(f) Reducing the instrumental photometric system to the standard Johnson-Cousins photometric system.

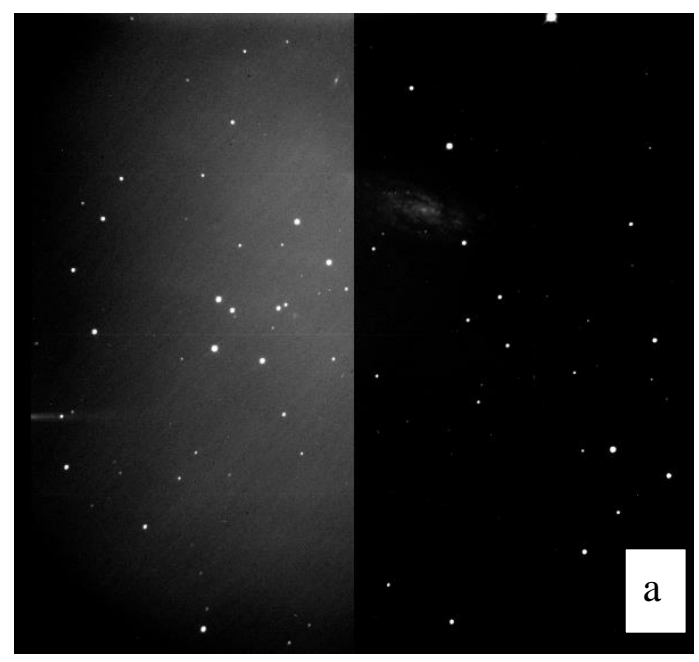

(g) Subtracting galaxy images taken in different filters in order to obtain colorindex map.

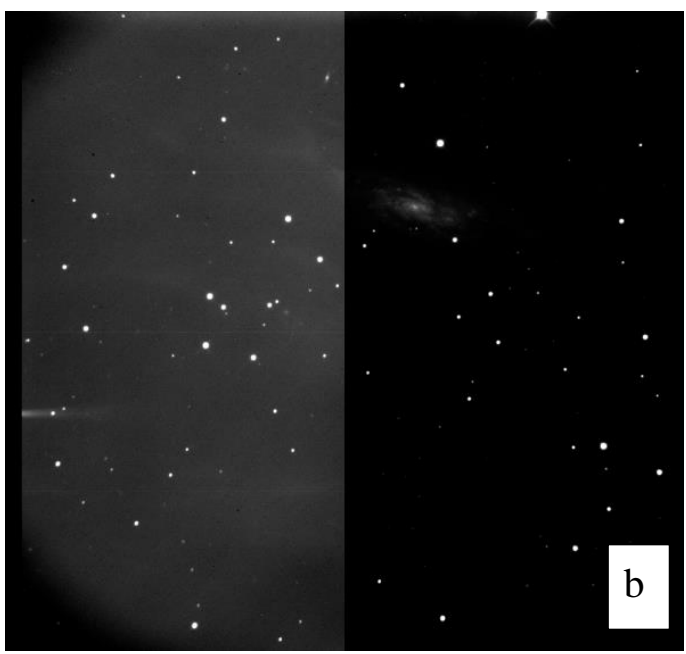

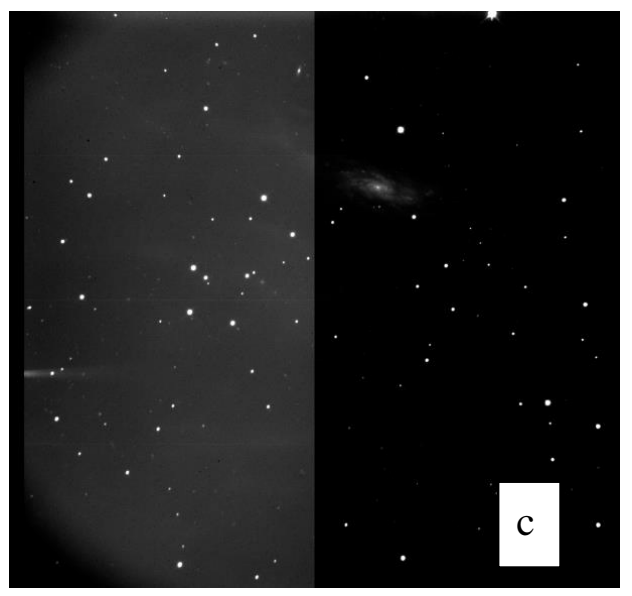

Fig.1: CCD image of Ic 467 before reduction (a) B filter (b) V filter (c) $R$ filter.

The output of the data reduction process is shown in Fig. 2. The results demonstrate clean background.

\section{Analysis of the photometric results}

Ic 467 has a large, bright core Fig.3 demonstrate $\mathrm{B}, \mathrm{V}$ and $\mathrm{R}$ the photometric sections along the major axis of Ic 467 passing through the galaxy's center. We estimate its diameter to be $\left(10^{\mathrm{n}}\right)$; the bulge can be seen out to $\left(20^{n}\right)$ from the center. The brightness falls off exponentially along the bar. The surface brightness of the bar in $\mathrm{V}$ filter is $\left(20.9 \pm 0.2 \mathrm{~m} / \operatorname{arcsec}^{2}\right)$. At the eastern edge of the bar, the surface brightness increases in $\mathrm{V}$ filter to $(20.8 \pm 0.1$ $\mathrm{m} / \operatorname{arcsec}^{2}$ ). Two bright spiral arms emerge from the edges of the bar. The surface brightness of the eastern and western arms in V filter are $\left(21.3 \pm 0.2 \mathrm{~m} / \operatorname{arcsec}^{2}\right)$ and $(21.5$ $\pm 0.2 \mathrm{~m} / \operatorname{arcsec}^{2}$ ) respectively. The surface brightness of the eastern arms in $\mathrm{V}$ filter is brighter than the western arm. The maximum surface brightness of the core in $\mathrm{B}, \mathrm{V}$ and $\mathrm{R}$ filters are $17.3 \mathrm{~m} / \operatorname{arcsec}^{2}$, and 
$18.3 \mathrm{~m} / \quad \operatorname{arcsec}^{2}$ and $18.3 \mathrm{~m} / \operatorname{arcsec}^{2}$ respectively. Fig. 4 shows the surface plot texture of Ic 467.

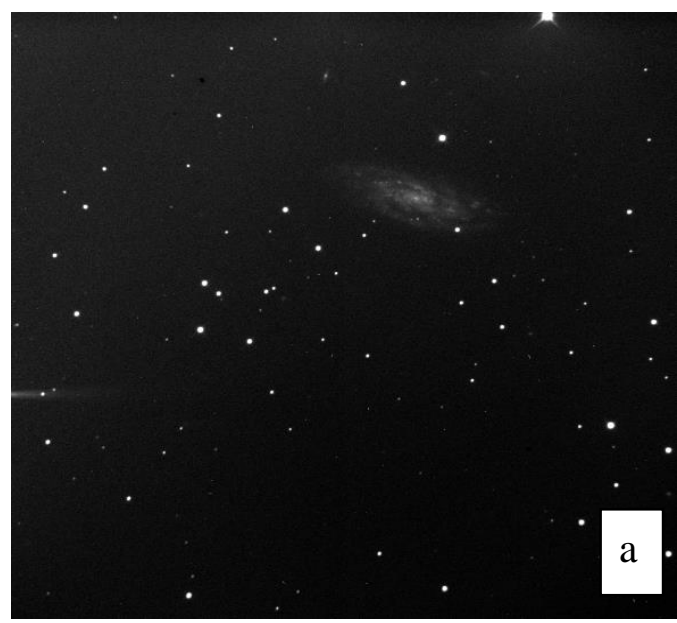

We are able to study the dependence of the isophote ellipticity and position angle on distance from the center of the galaxy.
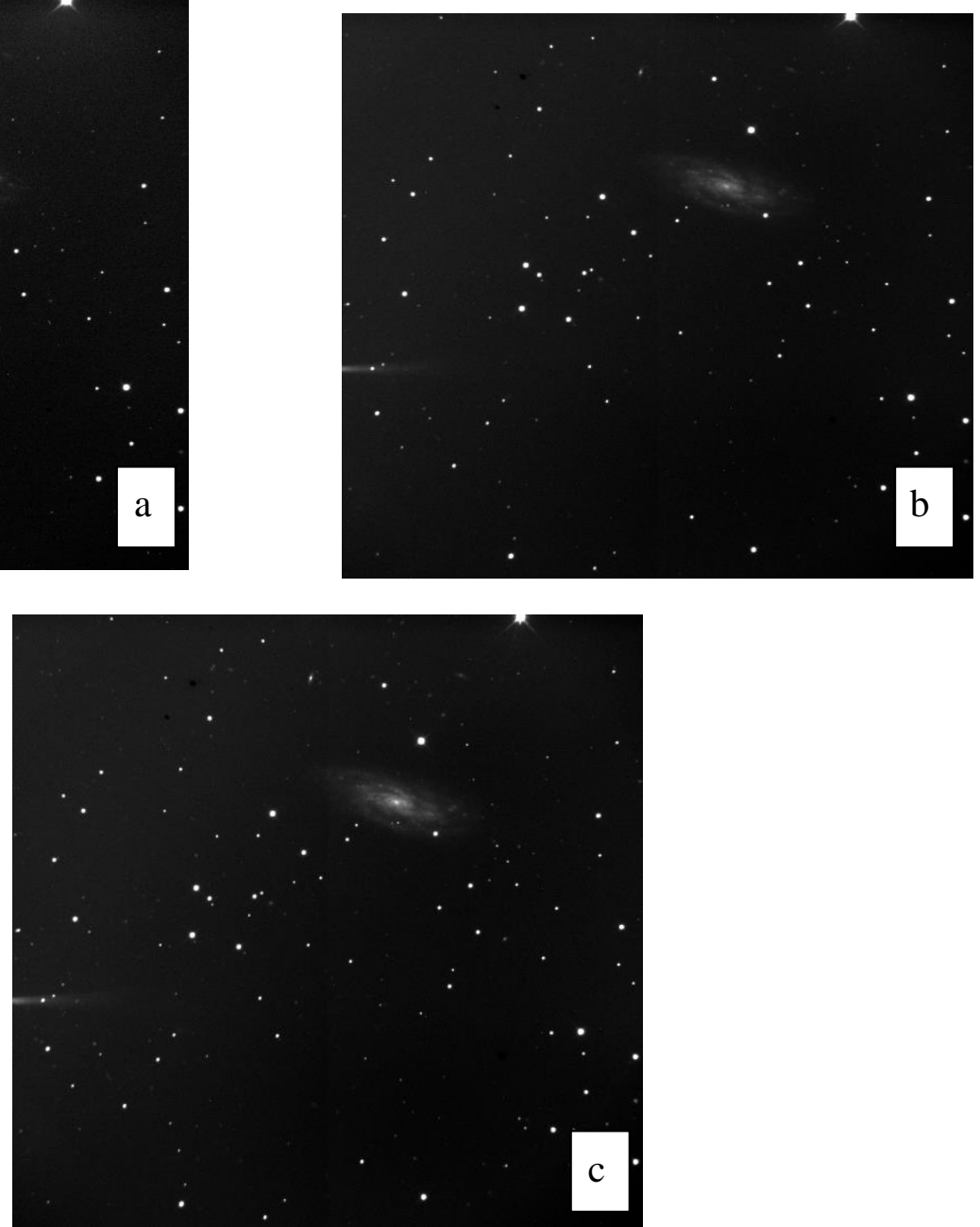

Fig.2: CCD image of Ic 467 after reduction (a) B filter (b) V filter (c) R filter.

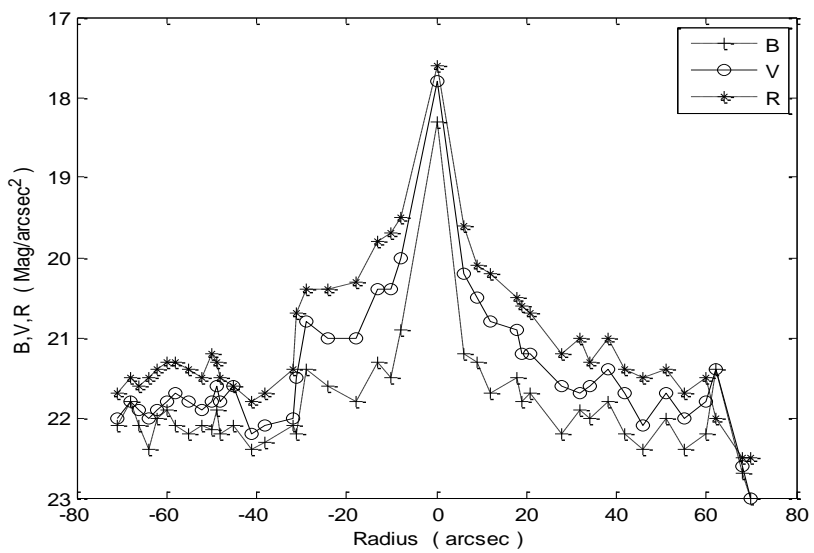

Fig.3: Photometric profiles along the major axis of Ic 467. 


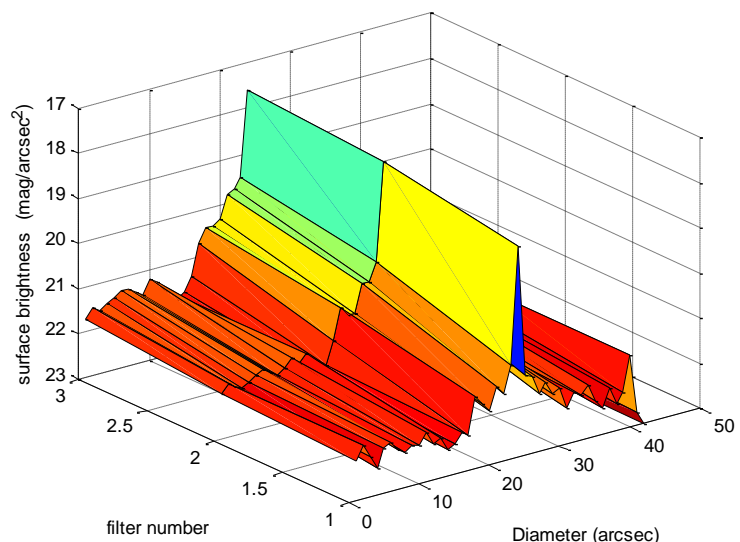

Fig.4: Surface plot of texture brightness of Ic 467

Fig.(5a) and (5b) illustrate the ellipticity and position angle of the isophotes as a function of the distance from the center of the galaxy Ic 467. In the central regions $r<30$ ", the isophotes are essentially circular $(\mathrm{e}=0.10 \pm$ 0.05 ), and the position angle is $\mathrm{PA}=95^{\circ}$ $\pm 10^{\circ}$. For more distant regions, the $\mathrm{e}(\mathrm{r})$ and PA(r) dependences are affected by the bright spiral arms. The isophote ellipticity increases sharply at $\mathrm{r}>35^{\prime \prime}$ then decreases slightly at $r>45 "$. The position angle of the galaxy increases smoothly at $r>40 "$.

Fig.6 represents (B-V) and (V-R) color indices map along the major axis of Ic 467. The galactic nucleus is very blue, with color indices $\mathrm{B}-\mathrm{V}=1.1 \mathrm{mag}, \mathrm{V}-\mathrm{R}=0.7 \mathrm{mag}$. One peculiarity of the galaxy is the presence near the center of a very blue object a giant star forming region. The bulge is the reddest region of the galaxy $\mathrm{B}-\mathrm{V}=0.6 \mathrm{mag}$, $\mathrm{V}-\mathrm{R}=0.4 \mathrm{mag}$. The color indices of the eastern bar are $\mathrm{B}-\mathrm{V}=0.71, \mathrm{~V}-\mathrm{R}=0.49$ and for the western bar are $\mathrm{B}-\mathrm{V}=0.4 \mathrm{mag}, \mathrm{V}-\mathrm{R}=0.3$ mag. The galaxy is symmetric the color indices of the eastern arm are $\mathrm{B}-\mathrm{V}=0.4 \mathrm{mag}$, $\mathrm{V}-\mathrm{R}=0.29 \mathrm{mag}$ and for the western arm are $\mathrm{B}-\mathrm{V}=0.4 \mathrm{mag}, \mathrm{V}-\mathrm{R}=0.1 \mathrm{mag}$.
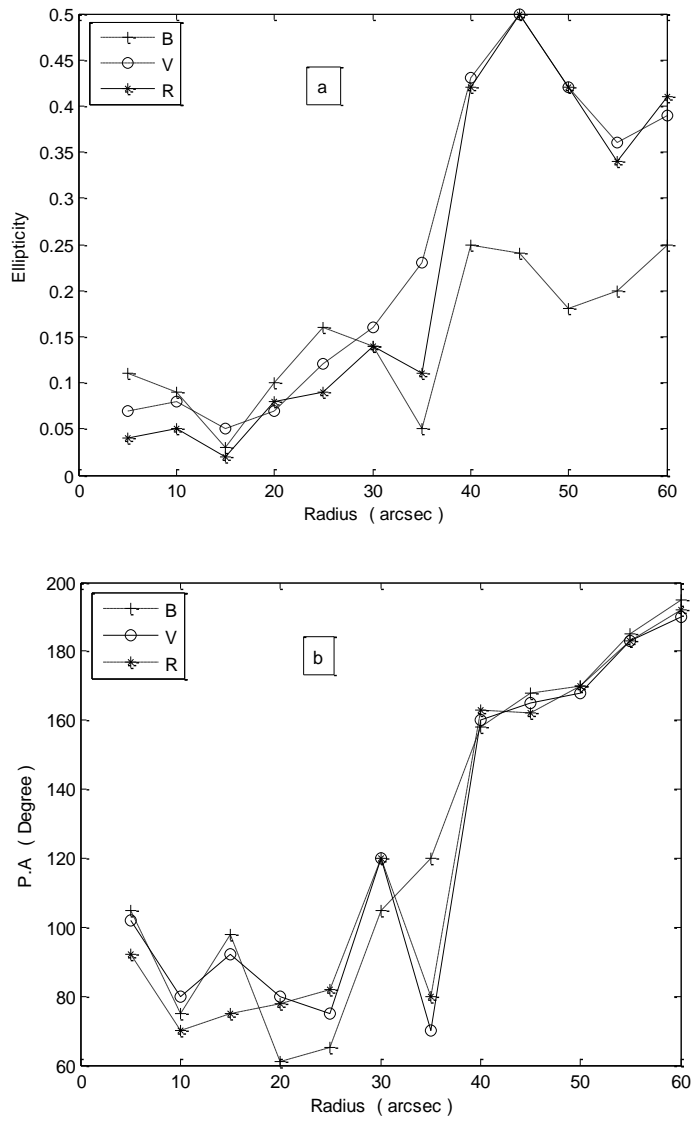

Fig.5: (a) Isophote ellipticity (b) position angle of the galaxy $P$. A as a function of the distance $r$ to the center of Ic 467 in $B, V$ and $R$ filters.

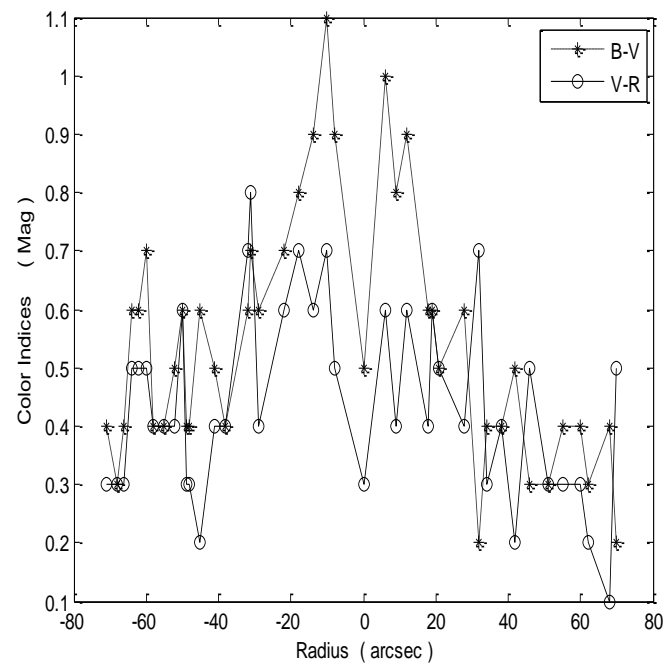

Fig.6: Profiles of the color indices along the major axis of the galaxy Ic 467. 


\section{Conclusions}

The surface photometry that carried out at Kottamia astronomical observatory using $1.88 \mathrm{~m}$ optical telescope shows that Ic 467 is one of the bluest galaxies in its class, and star formation has actively occurred. The star formation rate in the nucleus and spiral arms has remained virtually constant in time (as we can conclude from their positions in two-color diagrams). This means that either the efficiency of star formation has increased in time in these regions or there is a constant input of gas to the nucleus and spiral arms of the galaxy.

\section{References}

[1] A. S. Gusev, D. S. S. Kaisin, Astronomy Reports, 46, 9 (2002) 712-720.

[2] J. Schombert, A. K. Smith, in prep. arXiv:1107.1728v1 [astro-ph.IM] 8 Jul 2011.
[3] M. Spano, M. Marcelin, P. Amram, C. Carignan, Epinat, Mon. Not. R. Astron. Soc., 383 (2008) 297.

[4] A. M. I. Osman, "Astronomy for Developing Countries", 24th meeting of the IAU, Special Session, Manchester, England, August (2000).

[5] D. M. Elmegreen and B. G. Elmegreen, Astrophys. J., 314 (1987) 3.

[6] A. Landolt, Astron. J., 104 (1992) 340.

[7] H. A. Sinan, College of Education Magazine, University of Al-Mustansiryah, Iraq, 4 (2013) 876-882.

[8] H. M. Herna'ndez-Toledo, J. ZendejasDomI' nguez, and V. Avila-Reese. The Astronomical Journal, 134 (2007) 22862307.

[9] A. S. Gusev, A.V. Zasov, S. S. Kaisin, Astronomy Letters, 29, 6 (2003) 363-371. 